This item was submitted to Loughborough's Research Repository by the author.

Items in Figshare are protected by copyright, with all rights reserved, unless otherwise indicated.

\title{
Production of streptomycin from chitin using Streptomyces griseus in bioreactors of different configuration
}

PLEASE CITE THE PUBLISHED VERSION

PUBLISHER

(c) Elsevier

LICENCE

CC BY-NC-ND 4.0

REPOSITORY RECORD

Meanwell, Richard J.L., and Gilbert Shama. 2008. "Production of Streptomycin from Chitin Using Streptomyces Griseus in Bioreactors of Different Configuration”. figshare. https://hdl.handle.net/2134/3467. 
This item was submitted to Loughborough's Institutional Repository by the author and is made available under the following Creative Commons Licence conditions.

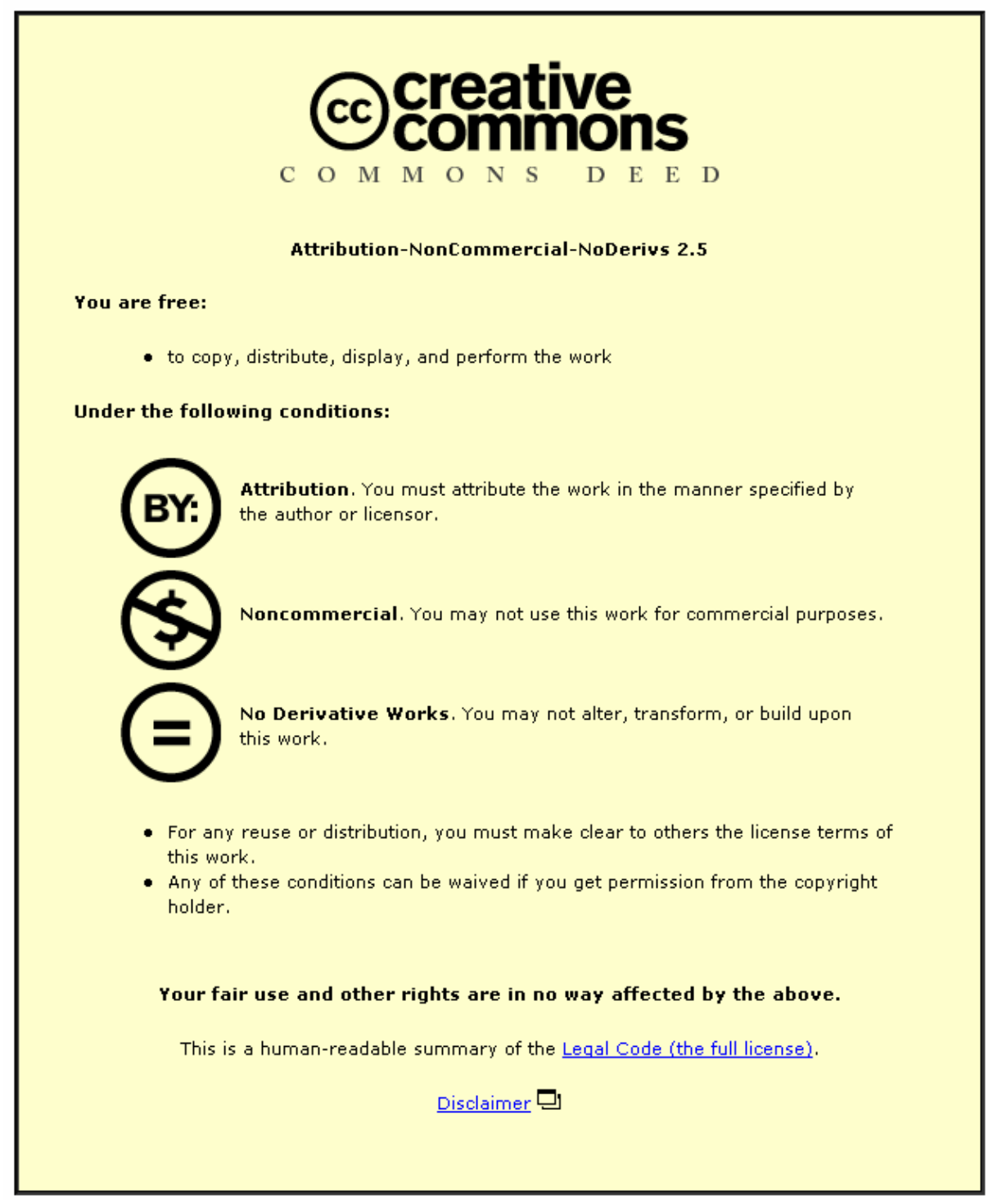

For the full text of this licence, please go to: http://creativecommons.org/licenses/by-nc-nd/2.5/ 


\title{
Production of Streptomycin from Chitin using Streptomyces griseus in Bioreactors of Different Configuration
}

\author{
Richard J.L. Meanwell and Gilbert Shama* \\ Department of Chemical Engineering, Loughborough University, Loughborough, \\ Leics., LE11 3TU, UK
}

\begin{abstract}
Streptomyces griseus was cultured in three different bioreactors in a medium containing chitin flakes. When a conventional bioreactor stirred by two sets of Rushton impellers and operated at high speed was used, the yield of streptomycin (3.1 $\mathrm{mg} / \mathrm{l}$ ) was the highest observed and occurred at approximately 500 hours. Cultivation of S. griseus in a bioreactor stirred at low speed by a U-shaped paddle resulted in a lower yield of streptomycin $(1.8 \mathrm{mg} / \mathrm{l})$ but this was achieved in a shorter period of time (400 hours). Increasing the concentration of chitin from 5 to $10 \% \mathrm{w} / \mathrm{v}$ had no significant effect on either of these two parameters. The use of a novel vertical basket bioreactor in which the chitin flakes were contained within a wire mesh basket and were gently fluidised by air, enabled comparatively high yields of streptomycin (2.8 $\mathrm{mg} / \mathrm{l})$ in the relatively short time of 300 hours.

Keywords: Chitin; Streptomyces; Streptomycin; Stirred Bioreactors; Vertical Basket Bioreactor

* Corresponding author. Fax: + 44 (0)1509 223923. E-mail address: g.shama@lboro.ac.uk.
\end{abstract}




\section{Introduction}

Commercial shellfish processing results in the worldwide generation of large tonnages of wastes (Chang et al., 2007). The quantities of waste produced are dependant both on the species of shellfish being processed and on the method of processing. For example, during shrimp processing in Norway some $40 \%$ of the weight of catches ultimately ends up as waste (Gildberg and Stenberg, 2001). Shellfish wastes are both highly perishable and highly polluting, but despite this, the commonest method of disposal is to discharge them to sea. However, as regulatory agencies tighten restrictions on such practices, alternatives to disposal are likely to assume greater importance.

Shellfish processing waste is a potentially rich resource of a number of useful compounds. Chief amongst these is chitin, the $\beta-(1 \rightarrow 4)$ linked homopolymer of $\mathrm{N}$ acetyl-D glucosamine, which is similar in structure to cellulose. Indeed, it has even been claimed that chitin is the second most abundant polymer on Earth after cellulose (Lee et al., 2007). The uses to which chitin can be put to are varied and growing in number. However, the principal applications appear to be in the food, medical, agricultural and environmental protection industries (Felse and Panda, 1999; Synowiecki and Al- Khateeb, 2003).

The traditional method of recovering chitin from shellfish waste is to deproteinise and demineralise it by a combination of acid and alkali treatments. However, the disadvantage of this form of treatment is that large volumes of liquid effluents are 
generated (Zakaria et al., 1998). One alternative to chemical treatment is to use micro-organisms to bring about rapid stabilisation of shellfish wastes.

Zakaria et al. (1998) demonstrated this principle with the lactic acid bacterium Lactobacillus paracasei in a horizontal basket bioreactor to produce a partially purified and stable chitin-rich solid product. Later, Yang et al., (2000) isolated a strain of Bacillus subtilis that was capable of deproteinising shellfish wastes. Cira et al., (2002) also using a lactic acid bacterium, achieved good chitin recovery using column bioreactors that could be scaled up to enable treatment of $30 \mathrm{~kg}$ batches. Beaney et al., (2005) recently provided validation of the fementative approach to chitin recovery by showing that the chitin produced by fermentation was directly comparable to that produced by chemical treatment on the basis of a number of parameters including the degree of acetylation.

A relatively new application for chitin is as a substrate in fermentation processes. In this instance the chitin serves as a source of energy and nutrients (principally $\mathrm{C}$ and N) to a microbial culture that is capable of producing one or more useful products. Mahadevan and Crawford (1997) cultured Streptomyces lydicus on chitin to produce chitinases with anti-fungal activity, whilst Trejo-Estrada et al., (2000) found that $S$. violaceusniger produced a number of polyene antifungal compounds related to guanidlyfungin A when grown on chitin. More recently, Wang et al. (2002) demonstrated that culture supernatants of a number of species of Monascus cultured on chitin all produced antifungal compounds. 
The streptomycetes are the most commercially important group of antibiotic producers and most species are chitinolytic (Schrempf, 2001). Streptomyces griseus is a particular case in point, and is used industrially to produce streptomycin. Streptomycin is a so-called 'broad spectrum antibiotic' i.e. active against both Gram positive and Gram negativeve bacteria, and belongs to the aminoglycoside group of antibiotics. First described by Selman Waksman in 1942, it is still in use today as an adjunct in the treatment of tuberculosis in humans (Di Perri and Bonora, 2004) but it also finds use as a veterinary drug (Jones et al., 2002).

In this work we compare streptomycin production from chitin in three bioreactors each of different configuration. The first of these was a conventional stirred bioreactor equipped with two sets of Rushton impellers, the second bioreactor was essentially a modification of the former in which one of the Rushton impellers was replaced by a U-shaped impeller and which was operated at low rotational speed. The third bioreactor investigated here was one of novel design and incorporated a static vertical basket in which the chitin was contained whilst being immersed in an aqueous salts medium. Air was passed directly into the basket so as to both provide oxygen to the culture of S. griseus, and to gently fluidize the chitin flakes. 


\section{Methods}

\subsection{Micro-organism \& Cultivation}

Streptomyces griseus (NCIMB 8136) was purchased in lyophilised form (NCIMB, Aberdeen, UK). Spores of the organism were maintained on sterile soil and were cultured on yeast malt extract agar (NCIMB Medium No. 29) at $28^{\circ} \mathrm{C}$ when required for bioreactor experiments. The liquid medium used for such experiments comprised flakes of chemically purified chitin (Sigma Aldrich plc, Poole, Dorset, UK) (at concentrations specified in the text and the following salts per litre of distilled water; $\mathrm{K}_{2} \mathrm{HPO}_{4}, 0.76 \mathrm{~g} ; \mathrm{KH}_{2} \mathrm{PO}_{4}, 0.3 \mathrm{~g} ; \mathrm{MgSO}_{4}, 0.5 \mathrm{~g} ; \mathrm{FeSO}_{4}, 0.01 \mathrm{~g} ; \mathrm{ZnSO}_{4}, 0.0018 \mathrm{~g}$; $\mathrm{MnCl}_{2}, 0.0016 \mathrm{~g}$. The $\mathrm{pH}$ of the medium was adjusted to 6.5 and was autoclaved at $121^{\circ} \mathrm{C}$ for 20 minutes. Inoculum for bioreactors comprised $100 \mathrm{ml}$ of culture grown for 48 hours in an incubator-shaker at $100 \mathrm{rpm}$ and $28^{\circ} \mathrm{C}$.

\subsection{Streptomycin Determination}

The concentration of streptomycin was determined using a standard bioassay procedure (Anon., 1998) and was based on the inhibition of the growth of Bacillus subtilis (NCIMB 8054). The only variation introduced into the procedure was that the spores of $B$. subtilis necessary for seeding the agar were produced by the method of Harnulv and Snygg (1972). Culture broths were first filtered through $0.2 \mu \mathrm{m}$ Whatman cellulose nitrate membrane filters (Fisher Scientific, Loughborough, Leics.) and subsequently using 2 kDa membranes (Dow Danmark A/S, Nakskov, Denmark). Aliquots of filtered culture broths $(150 \mu \mathrm{L})$ were added to wells cut into agar plates 
which were incubated for 48 hours at $30^{\circ} \mathrm{C}$. Zones of inhibition were measured using Vernier callipers. All determinations were performed in duplicate.

A standard calibration curve was obtained using aqueous solutions of streptomycin (Sigma Aldrich plc).

\section{$2.3 \mathrm{CO}_{2}$ Determination}

Gas samples were taken from the air exit line of the bioreactor using a gas-tight syringe and directly injected into a gas chromatograph (Model 104, Pye Unicam, Cambridge, UK) equipped with a thermal conductivity detector operated at $180^{\circ} \mathrm{C}$. The glass chromatography column was $6 \mathrm{~mm}$ diameter and $175 \mathrm{~cm}$ long and was packed with Molecular Sieve 5A (Phase Separations, Deeside, Clwyd, UK). All determinations were performed in duplicate.

\subsection{Biomass Determination}

Samples $(10 \mathrm{ml})$ were centrifuged at $8,600 \mathrm{x}$ g for 5 minutes at $10^{\circ} \mathrm{C}$. The supernatants were discarded and the cell pellet re-suspended in an equal volume of distilled water and re-centrifuged as above. The biomass was dried at $105^{\circ} \mathrm{C}$ overnight and weighed. All determinations were performed in duplicate.

\subsection{Bioreactors}

\subsubsection{Stirred Bioreactors}


A standard stirred 2-litre glass bioreactor vessel (LH Engineering Co. Ltd., Stoke Poges, Bucks, UK) was used. The working volume was 1.5 litres. This was used for experiments as originally supplied with two sets of Rushton impellers (Dia. $5 \mathrm{~cm}$ ) and four equidistantly spaced baffles or by removing the baffles and the lower of the 2 Rushton impellers and replacing it with a U-shaped paddle agitator. The latter was 23 $\mathrm{cm}$ wide and $18 \mathrm{~cm}$ high and fabricated from stainless steel strips $1 \mathrm{~cm}$ wide. In both cases the air flowrate to the sparger was $100 \mathrm{ml} / \mathrm{min}$.

\subsubsection{Vertical Basket Bioreactor}

This comprised a cylindrical wire frame of height $105 \mathrm{~cm}$ and diameter $65 \mathrm{~cm}$ fabricated around a hollow central support member and held in place by circular end plates. Wire mesh $(0.2 \mathrm{~mm}$ aperture $)$ was wound round the frame to create a chamber into which the chitin could be contained. The base plate was fabricated from sheet steel fitted with a wire mesh plate $1 \mathrm{~cm}$ above it. Air was admitted into the basket via the hollow central support. The air emerged from four $0.5 \mathrm{~mm}$ diameter holes at the base of the support and below the wire mesh and flowed upwards in the form of fine bubbles through the basket. The liquid working volume was 1.0 litre. An air flowrate of $100 \mathrm{ml} / \mathrm{min}$ was found to be sufficient to bring about gentle fluidisation of the chitin flakes contained in the chamber. In addition, two aerators were installed outside of the basket to both further aerate the liquid medium and to provide agitation; the flowrate to each of these aerators was $150 \mathrm{ml} / \mathrm{min}$. The total liquid volume was 1 litre. The mass of chitin flakes added to the basket was $35 \mathrm{~g}$. 


\section{Results and discussion}

\section{1 $\mathrm{CO}_{2}$ Evolution and Biomass Concentration}

Figure 1 shows the time course of fermentation for S. griseus cultured in yeast-malt extract fermentation medium devoid of chitin and conducted in a bioreactor equipped with two sets of Rushton impellers. The $\mathrm{CO}_{2}$ evolution curve and the biomass concentration curve correlate quite closely with one another and both reach a maximum at approximately 50 hours. The streptomycin peak $(3.0 \mathrm{mg} / \mathrm{l})$ occurred at approximately 200 hours when $\mathrm{CO}_{2}$ evolution had markedly declined.

The close correlation between $\mathrm{CO}_{2}$ and biomass concentrations provides confirmation that the latter may be used as a reliable indicator of growth. Desgranges et al. (1991) reached the same conclusion when they evaluated a number of indirect methods for measuring biomass concentration including $\mathrm{CO}_{2}$ evolution, and glucosamine and ergosterol assays. This was a useful finding as subsequent fermentations were carried out in the presence of solid chitin which effectively prevented the direct determination of cell dry weight. This occurred because as the streptomycete grew, its filaments formed very close associations with the chitin flakes and it was not possible to the two in order to assay them. This was revealed in scanning electron micrographs (Meanwell, 2004).

\subsection{Chitin Fermentation in a Conventional Stirred Bioreactor}

Figure 2 shows the results obtained during cultivation of S. griseus in a $5 \% \mathrm{w} / \mathrm{v}$ suspension of chitin using the bioreactor equipped with two Rushton turbines. $\mathrm{CO}_{2}$ 
evolution appears relatively constant for approximately the first 350 hours after which it rose relatively sharply. The maximum streptomycin concentration of $3.1 \mathrm{mg} / 1$ occurred at 375 hours, this was later than in the yeast - malt extract fermentation medium but was marginally greater than the maximum attained in that fermentation. The $\mathrm{pH}$ change over the entire time course of the fermentation amounted to less than 0.4 units and suggests that the chitin provided some buffering capacity.

The reduced $\mathrm{CO}_{2}$ evolution over the first 350 hours compared to the fermentation conducted in the absence of chitin is an indication of growth suppression and conditions inimical to growth may have been created by the high speed $(375 \mathrm{rpm})$ at which it was necessary to operate the impeller. High-speed operation could be envisaged as generating abrasive forces between the chitin particles and the streptomycete filaments and these could have damaged the growing tips of the filaments. Evidence of this was obtained by Meanwell (2004) who subjected biomass from shake flask- grown S. griseus to agitation in the presence and absence of chitin under non-growth conditions. By measuring the mean length of streptomycete filaments using image analysis software, he was able to show that rapid agitation resulted in a reduction in filament length of $75 \%$ but addition of only $0.4 \% \mathrm{w} / \mathrm{v}$ chitin reduced this still further to only $2 \%$ of the original length. Operation at speeds lower than $375 \mathrm{rpm}$ was found not to be practical as the chitin settled out of suspension.

\subsection{Detection of Anti-microbial Compounds other than Streptomycin}


An unexpected feature of this fermentation experiment was the detection of small quantities of anti-microbial compounds at an early stage of growth. These had a maximum inhibitory affect against the assay bacterium (B. subtilis) equivalent to 0.8 $\mathrm{mg} /$ litre of streptomycin, however, the concentration of these compounds declined rapidly and they were not observed after the first 50 hours. It was found that these compounds could be removed by filtering the culture broths through a $2 \mathrm{kDa}$ membrane. GC and HPLC assays were employed in the early phases of fermentation in order to eliminate definitively the presence of streptomycin and whilst none was detected, the tests were too insensitive for all but the highest concentrations of streptomycin encountered in this work (Meanwell, 2004). Reliance had therefore to be placed on the bioassay as the most sensitive method for detecting streptomycin. Identifying the compounds was beyond the scope of the present work, however, their appearance in early idiophase was very strongly indicative that they were not secondary metabolites. In utilising chitin S. griseus synthesises enzymes capable of dissimilating the polymeric substrate (Berger and Reynolds, 1958). These chitinases have themselves been shown to be potent fungal inhibitors (Wang et al., 2002; Wang et al., 2005) but would have no effect on the assay bacterium (B. subtilis) used here. The action of chitinases is to generate chitin oligomers which are known to be inhibitory towards certain bacteria. Indeed, Omura, et al., (2003) showed that chitin oligomers were capable of inhibiting the growth of B. subtilis. Kendra et al., (1989) claimed that oligomers of chain length of 7 and 8 units caused the greatest inhibitory effect. The retention characteristics of the filter through which fermentation samples were filtered in this work was selected specifically to remove compounds equivalent to the molecular weight of such oligomers. However, the conditions generated inside the bioreactor by operating the impellers at high speed may have caused it to produce 
an altered pattern of chitin oligomers of low chain length that were not retained by the filter. As a consequence of the appearance of these low molecular weight compounds in the early phases of fermentation all titres measured by bioassay in this and subsequent fermentations are reported as 'apparent streptomycin' titres.

\subsection{Chitin Fermentation in a Bioreactor Equipped with a U-shaped Paddle}

The growth of S. griseus on $5 \% \mathrm{w} / \mathrm{v}$ chitin using the bioreactor equipped with a Ushaped impeller is shown in Figure 2. The use of the U-shaped paddle resulted in the maintenance of chitin flakes in suspension at the much lower rotational speed of 55 rpm. $\mathrm{CO}_{2}$ evolution peaked at about 300 hours and was considerably higher than was achieved in the bioreactor equipped with twin Rushton impellers indicating that growth of the streptomycete was less affected by the U-shaped impeller. Moreover, maximum streptomycin concentration occurred at approximately 400 hours - earlier than was achieved in the bioreactor equipped with Rushton impellers. Interestingly, the maximum titre achieved $(1.8 \mathrm{mg} / \mathrm{l})$ was below the value of $3.0 \mathrm{mg} / \mathrm{l}$ reached in the previous fermentation. Also significantly, no compounds interfering with the bioassay were detected in the early phases of growth.

If the increased $\mathrm{CO}_{2}$ evolution in the bioreactor equipped with the U-shaped paddle may be taken as an indication of reduced physical stresses on the growing S. griseus culture, such conditions would appear to be incompatible with high antibiotic production. Evidence of the beneficial effects of stress as a stimulant of antibiotic production exists in previous work: Nakata et al., (1999) showed that the imposition of heat, salt or ethanol stresses stimulated antibiotic production by Pseudomonas 
fluorescens. Similarly, Gao et al., (2001) cultured E. coli in a rotating wall bioreactor under low physical stress conditions and obtained less microcin antibiotic than when the bacterium was cultured in a shake flask where the stresses were greater.

The use of the U-shaped impeller enabled the concentration of chitin to be doubled to $10 \% \mathrm{w} / \mathrm{v}$ (Figure 3 ) without necessitating a need to increase the speed of rotation above $55 \mathrm{rpm}$. At such high concentrations of chitin only inadequate mixing was achieved with the Rushton impellers and chitin was observed to settle out of suspension. The maximum streptomycin titre $(2.1 \mathrm{mg} / \mathrm{l})$ was achieved at approximately 385 hours and the maximum $\mathrm{CO}_{2}$ evolution at approximately 310 hours. Whilst the titre was comparable to the results achieved at the lower chitin concentration of $5 \%$, the higher chitin concentrations would result in greater overall streptomycin recovery.

\subsection{Chitin Fermentation in a Vertical Basket Bioreactor}

The time course of fermentation using the vertical basket bioreactor is shown in Figure 3 . The charge of chitin added to the basket was equivalent to a concentration inside the basket equivalent to $10 \% \mathrm{w} / \mathrm{v}$. Under these conditions $\mathrm{CO}_{2}$ evolution peaked at approximately 175 hours whilst the maximum concentration of streptomycin was achieved shortly before 300 hours had elapsed; both these events occurred earlier than when the stirred bioreactors were used. The maximum streptomycin titre achieved $(2.8 \mathrm{mg} / \mathrm{L})$ was higher than that observed during 
fermentation of chitin at both 5 and $10 \% \mathrm{w} / \mathrm{v}$ in the bioreactor equipped with the Ushaped impeller.

\section{Conclusions}

Solid chitin flakes constituted a suitable substrate for the production of streptomycin by S. griseus. The highest yield of streptomycin was achieved under conditions in which the growing culture was subjected to relatively high abrasive forces in a conventional stirred bioreactor. The disadvantage of this method of cultivation was that the time necessary to achieve maximum yield was excessive. The use of the Ushaped paddle evidently reduced the physical stresses on the growing culture but resulted in relatively poor yields of streptomycin. The novel vertical basket bioreactor provided high yields of streptomycin at short fermentation times. Although the yields of streptomycin generated here were small in industrial terms, measures are available for increasing them quite considerably and improving the overall process economics. The use of industrial strains of S. griseus would produce many times the yields achieved here (Butler et al., 1996). In addition, although commercially purified chitin was used here for convenience, a lower cost substrate would be partially purified chitin generated by lactic acid fermentation of shellfish wastes (Zakaraia et al., 1998). Preliminary tests (Meanwell, 2004) have shown that streptomycin yields using this material are actually higher than for commercially available chitin, however, further work is needed using bioreactors to confirm this. 


\section{References}

Anon., 1998. Official Method 962.14, Chapter 33, pp 41-3, Association of Official Analytical Chemists (AOAC) Official Methods of Analysis, $16^{\text {th }}$ Edition. Volume 2, AOAC International, Gaithersburg, MD., USA.

Beaney, P., Lizardi-Mendoza J. and Healy M., 2005. Comparison of chitins produced by chemical and bioprocessing methods. J. Chem. Tech. Biotech. 80, 145-150.

Berger, L.R. and Reynolds, D.M., 1958. The chitinase system of a strain of Streptomyces-griseus, Biochim. Biophys. Acta, 29, 522-534.

Butler, P.R., Brown, M., and Oliver, S.G., 1996. Improvement of antibiotic titres from Streptomyces bacteria by interactive continuous selection. Biotech. Bioeng. 49, 185 196.

Chang, W.T., Chen, Y.C. and Jao C.L. 2007. Antifungal activity and enhancement of plant growth by Bacillus cereus grown on shellfish chitin wastes. Biores. Technol. 98, 1224-1230.

Cira, L.A., Huerta, S., Hall, G.M. and Shirai, K., 2002. Pilot scale lactic acid fermentation of shrimp wastes for chitin recovery, Proc. Biochem. 37, 1359-1366. 
Desgranges, C., Vergoignan, C., Georges, M., and Durand, A., 1991. Biomass estimation in solid state fermentation: I. Manual biochemical methods. Appl. Micro. Biotech. 35, 200-205.

Di Perri, G. and Bonora, S., 2004. Which agents should we use for the treatment of multidrug-resistant Mycobacterium tuberculosis? J. Antimicrobial Chemo. 54, 593602.

Felse, P.A. and Panda, T., 2000. Production of microbial chitinases - A revisit. Bioproc. Eng. 23, 127-134.

Gao, Q., Fang, A., Pierson, D.L., Mishra, S.K. and Demain, A.L., 2001. Shear stress enhances microcin $\mathrm{B} 17$ production in a rotating wall bioreactor, but ethanol stress does not. Appl. Micro. Biotech. 56, 384-387.

Gildberg, A. and Stenberg, E.A., 2001. A new process for advanced utilisation of shrimp waste. Proc. Biochem. 36, 809-812.

Harnulv, B.G. and Snygg, B.G., 1972. Heat-resistance of Bacillus subtilis spores at various water activities. J. Appl. Bact. 35, 615 - 624 .

Jones, Y.E., Chappell, S., McLaren, I.M., Davies, R.H. and Wray, C., 2002. Antimicrobial resistance in Salmonella isolated from animals and their environment in England and Wales from 1988 to 1999 . Vet. Rec. 150, 649-654. 
Kendra, D.F., Christian, D., Hadwiger, L.A., 1989. Chitosan oligomers from Fusarium-Solani pea interactions, chitinase beta-glucanase digestion of sporelings and from fungal wall chitin actively inhibit fungal growth and enhance disease resistance. Physiol. Mol. Plant Path. 35, 215 - 230.

Lee, Y.S., Park, I.H., Yoo, J. S. Chung, S. Y. Lee, Y.C. Cho, S.C. Ahn, C.M. Kim and Choi, Y.L. 2007. Cloning, purification, and characterisation of chitinase from Bacillus sp. DAU101. Biores. Technol. 98, 2734-2741.

Mahadevan, B. and Crawford, D.L., 1997. Properties of the chitinase of the antifungal biocontrol agent Streptomyces lydicus WYEC108. Enz. Micro. Tech. 20, 489-493.

Meanwell, R.J.L., 2004. Streptomycin production from chitin using Streptomyces griseus, $\mathrm{PhD}$ Thesis, Loughborough University.

Nakata, K., Yoshimoto, A. and Yamada, Y., 1999. Promotion of antibiotic production by high ethanol, high $\mathrm{NaCl}$ concentration, or heat shock in Pseudomonas fluorescens S272. Biosci. Biotech. Biochem. 63, 293-297.

Omura, Y., Shigemoto, M., Akiyama, T, Saimoto, H., Shigemasa, Y., Nakamura, I., and Tshchido, T., 2002. Reexamination of antimicrobial activity of chitosan having different degrees of acetylation and molecular weights. Adv. Chitin Sci. 6, 273 - 274.

Schrempf, H., 2001. Recognition and degradation of chitin by streptomycetes, Antonie Van Leeuwenhoek Int. J. Gen. Mol. Micro. 79, 285-289. 
Synowiecki, J. and Al-Khateeb, N.A., 2003. Production, properties, and some new applications of chitin and its derivatives. Crit. Revs.Food Sci. Nutr. 43, 145-171.

Trejo-Estrada, S.R., Paszczynski, A. and Crawford, D.L., 1998. Antibiotics and enzymes produced by the biocontrol agent Streptomyces violaceusniger YCED-9. J. Ind. Micro. Biotech. 21, 81-90.

Wang, S.L., Yen, Y.H., Tsiao, W.J., Chang, W.T. and Wang, C.L., 2002. Production of antimicrobial compounds by Monascus purpureus CCRC31499 using shrimp and crab shell powder as a carbon source. Enz. Micro. Tech. 31, 337-344.

Wang, S.L., Yen, Y.H., Tzeng, G.C. and Hsieh, C., 2005. Production of antifungal materials by bioconversion of shellfish chitin wastes fermented by Pseudomonas fluorescens K-188. Enz. Micro. Tech. 36, 49-56.

Yang, J.K., Shih, I.L., Tzeng, Y.M. and Wang, S.L., 2000. Production and purification of protease from a Bacillus subtilis that can deproteinize crustacean wastes. Enz. Micro. Tech. 26, 406-413.

Zakaria, Z., Hall, G.M. and Shama, G., 1998. Lactic acid fermentation of scampi waste in a rotating horizontal bioreactor for chitin recovery. Proc. Biochem. 33, 1-6. 
Figure Captions

Figure 1 Cultivation of S. griseus in Yeast-Malt Extract Medium

$(\Delta)$ Streptomycin Titre $(\diamond) \mathrm{CO}_{2}(\square)$ Biomass. Error Bars represent SME.

Figure 2 Cultivation of S. griseus on Chitin Medium (5\% wt/v) in Bioreactors Equipped with Double Rushton Impellers or U-shaped Impeller.

( $\square)$ Streptomycin Titre U-Shaped Impeller, $(\times)$ Streptomycin Titre Ruston Turbines

$(\diamond) \mathrm{CO}_{2}$ Concentration U-Shaped Impeller, $(\triangle) \mathrm{CO}_{2}$ Concentration Rushton Turbines. Error Bars represent SME.

Figure 3 Cultivation of S. griseus on Chitin Medium (10\%w/v) in a Bioreactor Equipped with U-shaped Impeller and in a Vertical Basket Bioreactor

( $\square)$ Streptomycin Titre U-Shaped Impeller, $(\times)$ Streptomycin Titre Basket Bioreactor

$(\diamond) \mathrm{CO}_{2}$ Concentration U-Shaped Impeller, $(\triangle) \mathrm{CO}_{2}$ Concentration Basket

Bioreactor. Error Bars represent SME. 
Figure 1

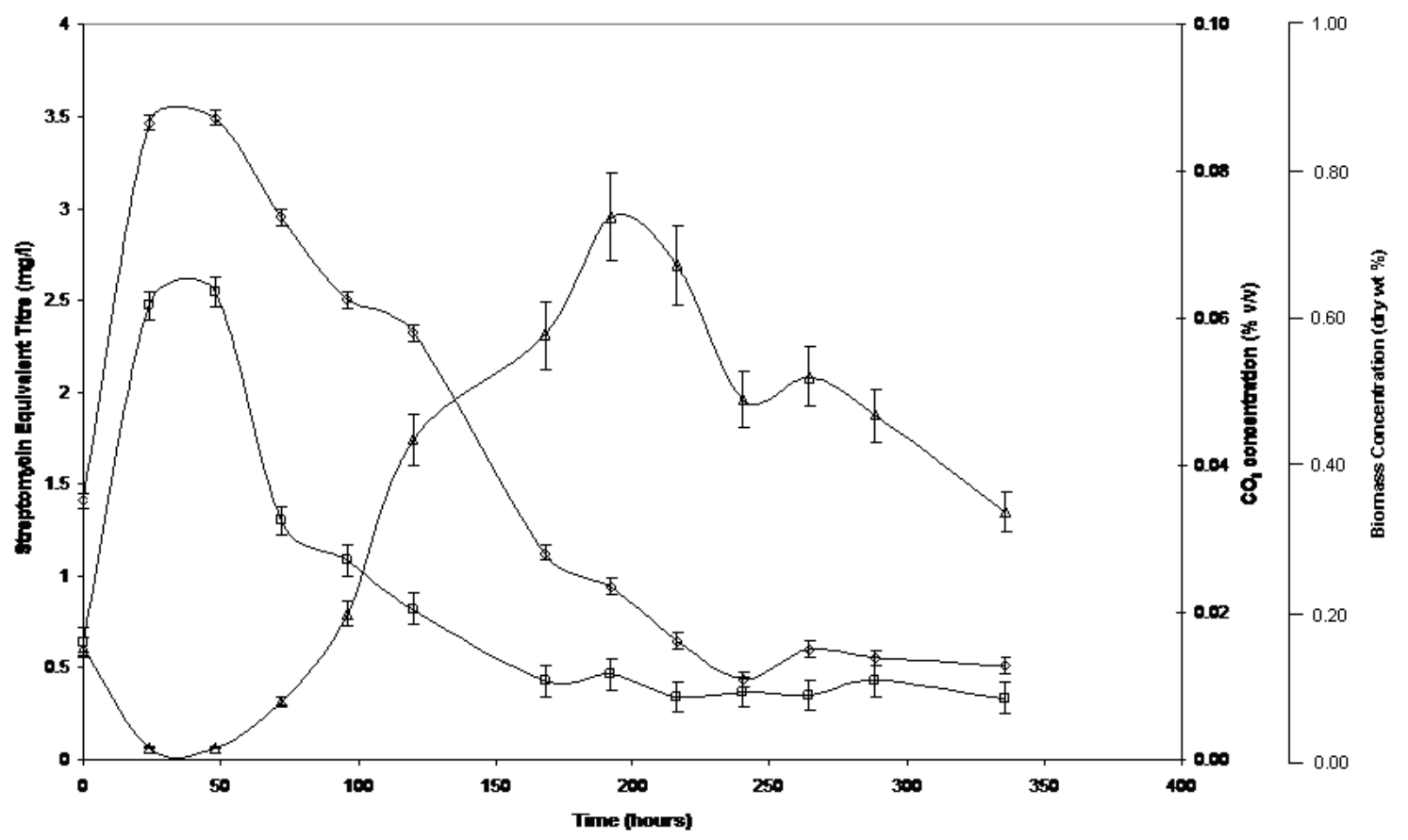


Figure 2

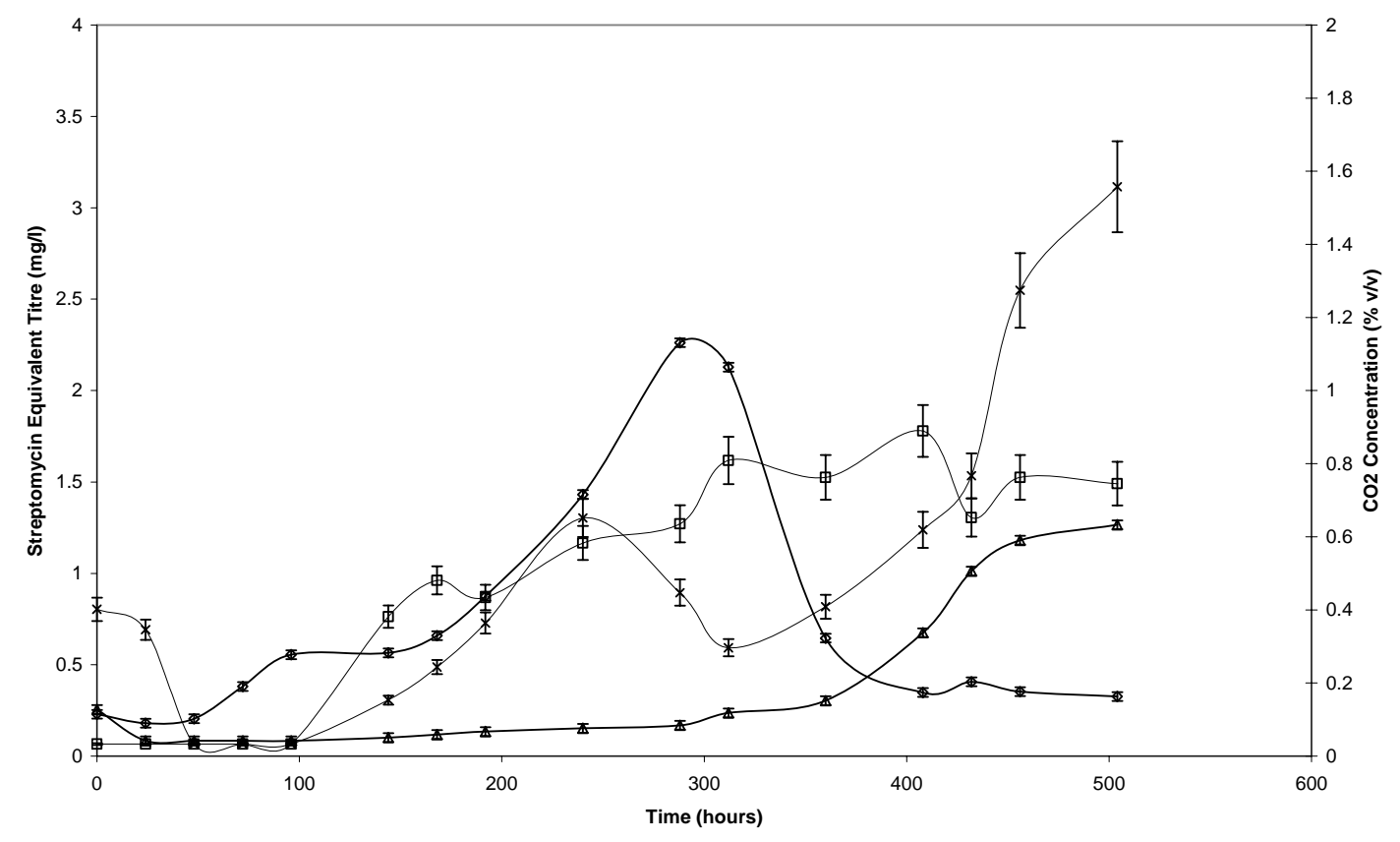


Figure 3

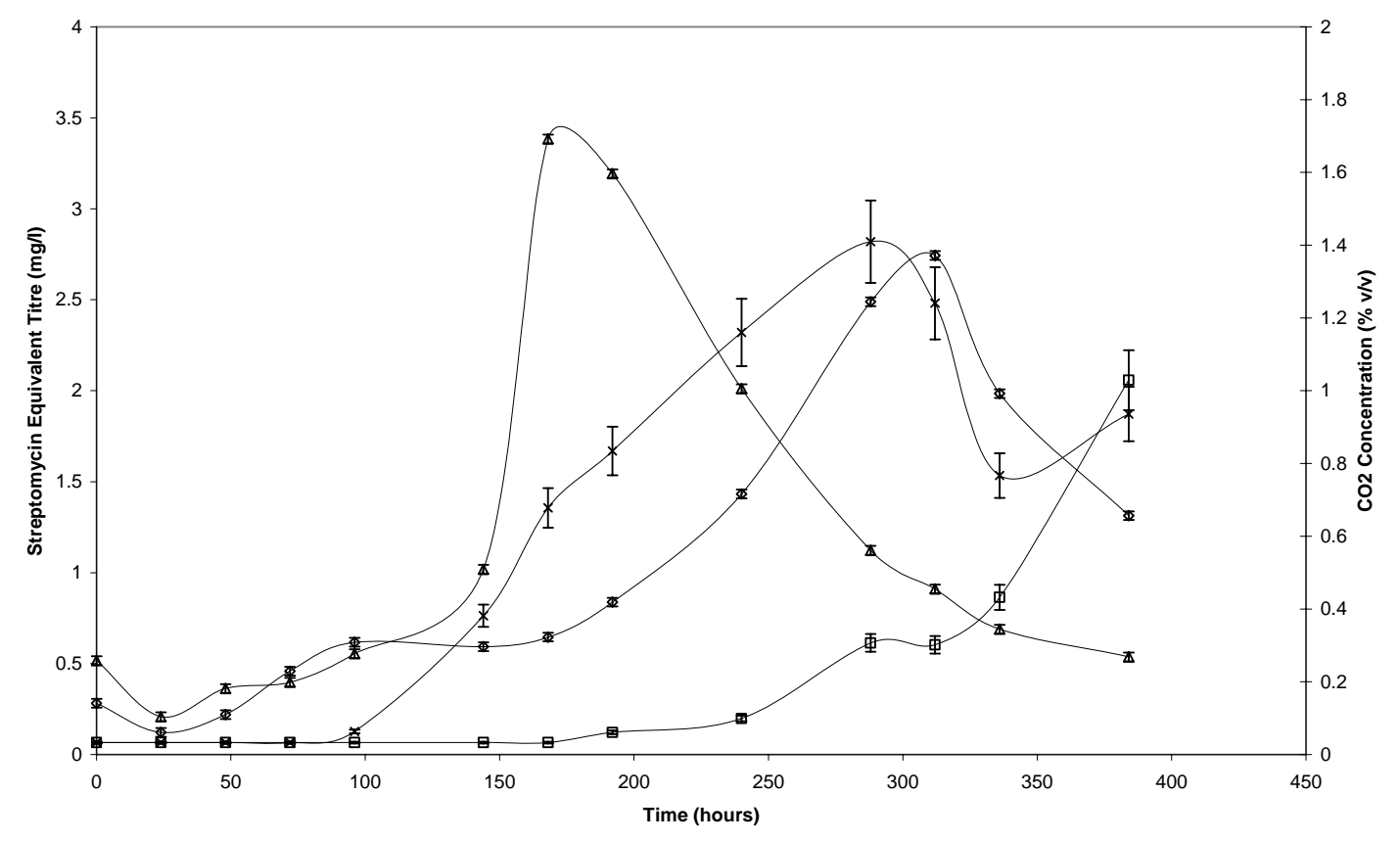

